This document is the accepted manuscript version of the following article:

Sibil1e, R., Mazzone, D. G., Ban, V., Mazet, T., \& François, M. (2018). Chemical1y-

controlled stacking of inorganic subnets in coordination networks: metal-organic magnetic

multilayers. Inorganic chemistry, 57(14), 8236-8240.

https://doi .org/10.1021/acs . inorgchem. 8 b00748

\title{
Chemically-Controlled Stacking of Inorganic Subnets in Coordination Networks: Metal-Organic Magnetic Multilayers
}

\author{
Romain Sibille,, Daniel G. Mazzone, ${ }^{1,2}$ Voraksmy Ban, ${ }^{3}$ Thomas Mazet ${ }^{4}$ and Michel François ${ }^{4}$ \\ ${ }^{1}$ Laboratory for Neutron Scattering and Imaging, Paul Scherrer Institut, 5232 Villigen PSI, Switzerland \\ ${ }^{2}$ National Synchrotron Light Source II, Brookhaven National Laboratory, Upton, New York 11973, USA \\ ${ }^{3}$ Laboratory for Condensed Matter Research, Paul Scherrer Institut, 5232 Villigen PSI, Switzerland \\ ${ }^{4}$ Université de Lorraine, CNRS, IJL, 5400o Nancy, France
}

Supporting Information

\begin{abstract}
Coordination Networks (CNs), such as for instance Metal-Organic Frameworks (MOFs), can turn into remarkable magnets, with various topologies of spin carriers and unique opportunities of cross-coupling to other functionalities. Alternatively, distinct inorganic subnetworks that are spatially segregated by organic ligands can lead to coexisting magnetic systems in a single bulk material. Here, we present a system of two $\mathrm{CNs}$ of general formula $\mathrm{Mn}\left(\mathrm{H}_{2} \mathrm{O}\right)_{x}\left(\mathrm{OOC}-\left(\mathrm{C}_{6} \mathrm{H}_{4}\right)_{y}-\mathrm{COO}\right)$. The compound with two water molecules and one aromatic ring $(x=2 ; y=1)$ has a single 2-dimensional magnetic subnet, while the material with $x=1.5$ and $y=2$ shows, additionally, another type of magnetic layer. In analogy to magnetic multilayers that are deposited by physical methods, these materials can be regarded as MetalOrganic Magnetic Multilayers (MOMMs), where the stacking of different types of magnetic layers is controlled by the choice of an organic ligand during the chemical synthesis. This work further paves the way towards organic-inorganic nanostructures with functional magnetic properties.
\end{abstract}

Coordination Networks (CNs), such as Metal-Organic Frameworks (MOFs), ${ }^{1}$ allow to combine magnetic and nonmagnetic entities that form hybrid magnetic materials ${ }^{2}$ - an approach that has been proved to be a reliable source of multifunctional materials. ${ }^{3}$ In particular, layered hybrid magnets, ${ }^{4}$ where a 2 -dimensional inorganic subnetwork alternates with organic pillar molecules along a stacking direction, have shown remarkable properties including giant hysteretic effects, ${ }^{5}$ high-temperature ferromagnetism, ${ }^{6}$ coexisting metallic conductivity and ferromagnetism, ${ }^{7}$ spin glass behavior $^{8}$ and switchable magnetism in response to external stimuli. ${ }^{9}$ However, hybrid layered materials (as well as MOFs in general) have only provided a limited number of examples in which different types of inorganic layers (subnetworks) coexist. ${ }^{10}$ Recently, two distinct magnetic ordering transitions have been observed in $\mathrm{Mn}\left(\mathrm{C}_{4} \mathrm{H}_{4} \mathrm{O}_{4}\right)$, a MOF where two types of inorganic layers alternate in an 'ABAB...' sequence." The layers are connected by succinate ligands, revealing a remarkable MOF that triggers an unusual physical behavior.
In this Article, we present a system that consists of two layered $\mathrm{CNs}$, in which the stacking of two types of inorganic subnetworks is controlled by the choice of the interlayer organic ligand. The formation of such Metal-Organic Magnetic Multilayers (MOMMs) further reveals the parallel between hybrid layered magnets and multilayers of inorganic materials deposited by physical methods, which have led to a multitude of technological applications.

Figure 1 shows a projection of the structures of the two CNs of general formula $\mathrm{Mn}^{2+}\left(\mathrm{H}_{2} \mathrm{O}\right)_{x}\left({ }^{-} \mathrm{OOC}-\left(\mathrm{C}_{6} \mathrm{H}_{4}\right)_{y}-\mathrm{COO}^{-}\right)$. The material with $x=2$ and $y=1$ has been reported earlier and is based on 1,4-benzenedicarboxylate (bdc). ${ }^{12}$ It features a single inorganic subnet labelled ' 1 '. The material with $x=1.5$ and $y=2$ is reported for the first time here and incorporates 4,4'-biphenyldicarboxylate (bpdc) molecules that separate two types of inorganic subnets; layer ' 1 ', as in the bdc-based material, and another type of layer labelled ' 2 '.

Both materials are formed at room-temperature by coprecipitation in water of the sodium(I) bdc or bpdc dicarboxylate with manganese(II) chloride. The $\mathrm{pH}$ value is determinant for their synthesis as it regulates the competition between the hydrated phases and the manganese(II) hydroxydicarboxylates, namely phases of general formula $\mathrm{Mn}^{2+}{ }_{2}\left(\mathrm{OH}^{-}\right)_{2}\left({ }^{-} \mathrm{OOC}-\left(\mathrm{C}_{6} \mathrm{H}_{4}\right)_{y}-\mathrm{COO}^{-}\right)$. For $y=1$ (bdc), the hydrate is best formed at a $\mathrm{pH}$ value of about 7.5, while the hydroxide is obtained as a pure phase for $\mathrm{pH}=8.5$ or slightly higher; intermediate $\mathrm{pH}$ values yield mixtures of the two phases. This global scenario is retained for $y=2$ (bpdc); with the range of optimal $\mathrm{pH}$ values shifted to higher values. The novel phase presented here was obtained by slow addition, using peristaltic pumps, of aqueous solutions of sodium hydroxide (o.1 M) and manganese(II) chloride $\left(5 \times 10^{-2} \mathrm{M}\right)$ into an aqueous reaction medium that contains the deprotonated ligand $(\mathrm{pH}=9)$. The solution of the metallic salt was incorporated at a constant rate over an hour, while the addition of the base was controlled by a loop that maintained the $\mathrm{pH}$ of the reaction medium at a value of 9.0 \pm o.1. The slow mixing yields a better crystallinity of the final product, whereas the control loop on the $\mathrm{pH}$ is crucial to avoid the formation of competing hydroxydes. The aqueous suspensions resulting 
from the room-temperature co-precipitation can be hydrothermally treated under various conditions of temperature (up to 180 Celsius) and time (up to several days). Within these ranges, the only effect of this optional hydrothermal treatment is to improve the crystallinity of the final product.

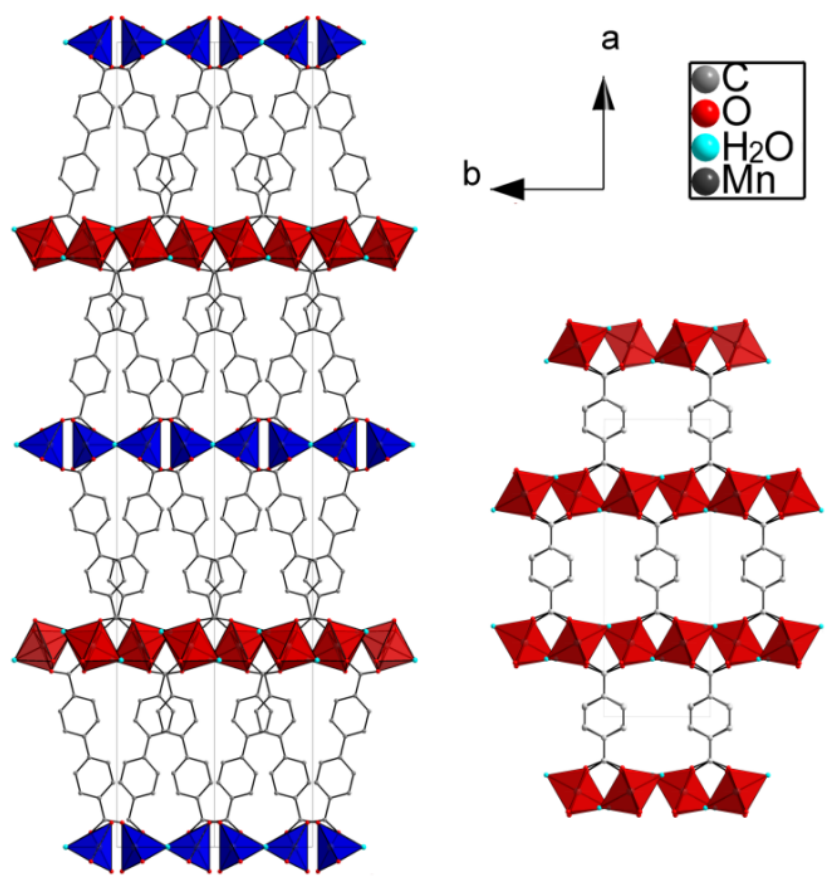

Figure 1. The system of controllable Metal-Organic Magnetic Multilayers (MOMMs) formed by $\mathrm{Mn}\left(\mathrm{H}_{2} \mathrm{O}\right)_{1.5}$ (bpdc) (left) and $\mathrm{Mn}\left(\mathrm{H}_{2} \mathrm{O}\right)_{2}$ (bdc) (right) Coordination Networks. Red and blue polyhedra correspond to the dihydrated ' 1 ' and monohydrated ' 2 ' types of layers, respectively.

$\mathrm{Mn}\left(\mathrm{H}_{2} \mathrm{O}\right)_{1.5}(\mathrm{bpdc})$ was obtained while we aimed to synthesize the bpdc analogue of $\mathrm{Mn}\left(\mathrm{H}_{2} \mathrm{O}\right)_{2}$ (bdc). The powder X-ray diffraction (PXRD) pattern is typical for layered hybrid materials (Figure $\mathrm{S}_{1}$ ), with a strong Bragg reflection centered on a low scattering angle. However, the solid does not crystallize into a monoclinic unit-cell that would simply account for the longer bpdc molecules. In contrast, the lattice parameter along the stacking direction, $\mathbf{a} \approx 53.35 \AA$, is remarkably large. The indexation into a monoclinic using the $\mathrm{X}$-Cell computer program $^{13}$ gives $\mathbf{b} \approx 6.49 \AA, \mathbf{c} \approx 7.41 \AA$ and $\beta \approx 90.45^{\circ}$, which is

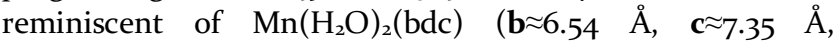
$\left.\beta \approx 97.85^{\circ}\right) .{ }^{12}$ The half-integer water stoichiometry of the novel material can be directly evaluated from a thermogravimetric analysis in air (Figure S2a), if manganese, bpdc and water molecules are considered as the constituents of the final product. The theoretical dehydration loss of $\mathrm{Mn}\left(\mathrm{H}_{2} \mathrm{O}\right)_{1.5}($ bpdc $)$ is $8.39 \%$, in excellent agreement with the experimental loss of $8.32 \%$. This process occurs well above 100 Celsius, which indicates coordinated water molecules. The decomposition of the organic ligand is observed around 350 Celsius and the total loss of $72.66 \%$ agrees well with the prediction for $\mathrm{Mn}\left(\mathrm{H}_{2} \mathrm{O}\right)_{1.5}($ bpdc $) \rightarrow 1 / 2 \mathrm{Mn}_{2} \mathrm{O}_{3}(75.48 \%$, residue checked by PXRD). Chemical analysis confirms the formula $\mathrm{C}_{28} \mathrm{H}_{22} \mathrm{Mn}_{2} \mathrm{O}_{11}$; Anal. (Calcd): C, 25.98 (26.71); O, 29.18 (29.68); $\mathrm{Mn}, 31.04$ (30.57). The crystal structure $\left(C_{2} / \mathrm{C}\right)$ of $\mathrm{Mn}\left(\mathrm{H}_{2} \mathrm{O}\right)_{1.5}($ bpdc $)$ was solved from synchrotron PXRD data by global optimization methods in the direct space, using the computer program Fox. ${ }^{14}$ The solution was obtained for a set of scatterers comprising one bpdc molecule in general position (8f), two $\mathrm{Mn}^{2+}$ ions occupying special positions $(\mathrm{Mn}(1)$ in $4 e$ and $\operatorname{Mn}(2)$ in $4 c)$, and two oxygen atoms accounting for the presence of two water molecules $\left(\mathrm{H}_{2} \mathrm{O}(1)\right.$ in $4 e$ and $\mathrm{H}_{2} \mathrm{O}(2)$ in $8 f$ ). The final Rietveld refinement of the synchrotron PXRD data was performed using Fullprof ${ }^{5}$ (Figure $\mathrm{S} 1$ ).

$\mathrm{Mn}\left(\mathrm{H}_{2} \mathrm{O}\right)_{1.5}$ (bpdc) is a novel $\mathrm{CN}$ featuring two types of inorganic subnets. Layer ' 1 ', represented in red (Figure 2), is the same as in $\mathrm{Mn}\left(\mathrm{H}_{2} \mathrm{O}\right)_{2}(\mathrm{bdc})$. It has the stoichiometry $1 \mathrm{Mn}(1)$ : $2 \mathrm{H}_{2} \mathrm{O}(1)$ and is composed of $\mathrm{MnO}_{6}$ octahedra where four of the six oxygen ligands are provided by the carboxylic functions of bpdc. Layer ' 1 ' forms a squared lattice of $\mathrm{MnO}_{6}$ octahedra interconnected through carboxylic bridges. The second layer ' 2 ' has the stoichiometry $1 \mathrm{Mn}(2): 1 \mathrm{H}_{2} \mathrm{O}(2)$ and is composed of $\mathrm{MnO}_{5}$ distorted trigonal bipyramids. These pentahedra form zigzag chains, via carboxylate bridges, running along the $\mathbf{c}$ direction. The chains are connected together via strong hydrogen bonds between the hydrogen atoms of $\mathrm{H}_{2} \mathrm{O}(2)$ and the acceptor oxygen atoms of the carboxylate groups in the adjacent chain. The donor-acceptor distance is $\approx 2.43 \AA$, which indicates bond energies in the range $60-170$ $\mathrm{kJ} . \mathrm{mol}^{-1}$. The existence of two crystallographicallyindependent water molecules is corroborated by the infrared spectrum in the region of interest where $\mathrm{O}-\mathrm{H}$ stretching vibrations are found (Figure $\mathrm{S}_{2} \mathrm{~b}$ ). The spectrum shows broad bands, as expected for $\mathrm{O}-\mathrm{H}$ groups that are involved in hydrogen bonds. The bands are centered around three distinct frequencies, which agrees with the existence of three donoracceptor distances around the oxygens of the water molecules $\left(\approx 2.69 \AA\right.$ and $\approx 2.81 \AA$ for $\mathrm{H}_{2} \mathrm{O}(1) ; \approx 2.43 \AA$ for $\left.\mathrm{H}_{2} \mathrm{O}(2)\right)$.
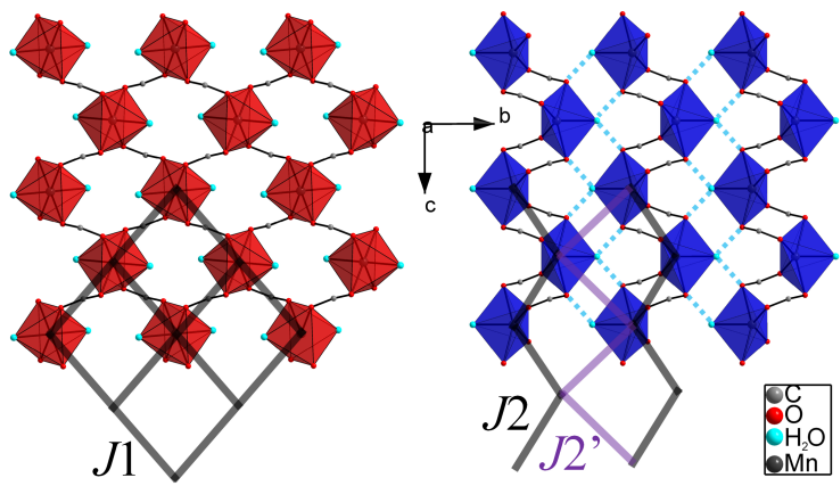

Figure 2. Magnetic subnetworks ' 1 ' (left) and '2' (right). ' 1 ' is approximated as a squared lattice with one exchange constant $J_{1}$. ' 2 ' is approximated as an array of zig-zag chains with one exchange constant $J 2$ along the chains, and an interchain path J2' through hydrogen bonds (blue dashed bonds).

The magnetic properties of both CNs were investigated by magnetization measurements recorded using a SQUID magnetometer (MPMS-XL, Quantum Design). The thermal dependence of the magnetization, $\mathrm{M}$, was probed in the temperature, $T$, range from 1.8 to $300 \mathrm{~K}$, under a moderate applied magnetic field, $\mu_{0} H$, of $0.1 \mathrm{~T}$. A simple adjustment of $H / M\left(\approx \chi^{-1}\right)$ vs. T to a Curie-Weiss law reproduces the experimental data down to a temperature of about $15 \mathrm{~K}$, for both $\mathrm{CNs}$ (Figure $3 \mathrm{a}$ and $3 \mathrm{~b}$ ). The experimental data significantly deviate from this simple behavior for lower temperatures, indicating the development of magnetic correlations. This is supported by the broad bump that is centered around $T \approx 6 \mathrm{~K}$ in the magnetic susceptibility $\chi(\approx M / H)$ of both compounds (Figure $3 \mathrm{c}$ and $3 \mathrm{~d}$ ). Such a behavior is typical for low- 
dimensional magnets. Further cooling below approximately 5 $\mathrm{K}$ reveals sharp anomalies attributed to actual magnetic phase transitions, vide infra.

As evidenced in Figure 2, the two types of inorganic layers can be described using simple topologies of first-neighbor magnetic interaction pathways. A nearly squared lattice with one exchange constant through carboxylate bridges $\left(J_{1}\right)$ is employed for the dihydrated layer ' 1 '. The intermetallic distance in ' 1 ' is $d_{\mathrm{Mn}(1)-\mathrm{Mn}(1) \approx 4.96} \AA$ and $d_{\mathrm{Mn}(1)-\mathrm{Mn}(1) \approx 4.92} \AA$ for the bdc- and bpdc-based materials, respectively. The monohydrated layer ' 2 ' requires a slightly more evolved model that corresponds to an array of chains with one intrachain interaction through carboxylate bridges $\left(J_{2}\right)$, and one interchain interaction through hydrogen bonds $\left(\mathrm{J}_{2}\right.$ '). In ' 2 ' the intermetallic distances are $d_{\operatorname{Mn}(2)-\operatorname{Mn}(2) \approx 4.48} \AA$ along $J 2$ and $d_{\mathrm{Mn}(2)-\mathrm{Mn}(2) \approx 5.43} \AA$ along $J_{2}$ '. We approximate all magnetic moments as isotropic (Heisenberg-like) spins, because $\mathrm{Mn}^{2+}$ is an orbitally non-degenerate cation $(\mathrm{L}=\mathrm{O})$ and is, therefore, only weakly affected by crystal-electric field effects.

Based on the above description of the two magnetic layers, we model their magnetic susceptibility using a $2 \mathrm{D}$ Heisenberg model $^{16}$ and a $\mathrm{iD}$ Fisher model ${ }^{17}$ with mean-field interchain interactions, respectively (see Figure 3 and Supporting Information). The experimental data arising from the bdcbased MOF are well fitted using the $2 \mathrm{D}$ Heisenberg model solely, yielding reasonable values for the exchange constant $\left(J_{1} / \mathrm{k}_{\mathrm{B}}=-3.356(1) \mathrm{K}\right.$ - indicating an antiferromagnetic interaction) and gyromagnetic factor $\left(g_{1}=\mathbf{2 . 1 1 1}(3)\right)$. The fit can be further improved by adding a mean-field correction that accounts for interlayer interactions (Figure $3 c$ ). In this case, we obtain $J_{1} / \mathrm{k}_{\mathrm{B}}=-\mathbf{3 . 2 4 3}(3) \mathrm{K}, g_{1}=\mathbf{2 . 2 0 9}(2)$, and the mean-field constant $J_{\text {interlayer }} / \mathrm{k}_{\mathrm{B}=-0.91(2)} \mathrm{K}$. In contrast, the magnetic susceptibility of the bpdc-based material is best fitted with equally-weighted contributions of ' 1 ' and ' 2 ' (Figure $3 \mathrm{~d}$ ). We find $J_{1} / \mathrm{k}_{\mathrm{B}}=-2.593(4) \mathrm{K}$ and $g_{1}=2.065(9)$ for the $2 \mathrm{D}$ Heisenberg layer ' 1 ', and $J_{2} / \mathrm{k}_{\mathrm{B}}=-2.35(2) \mathrm{K}, g_{2}=2.373(9)$ and $J 2$ ' $/ \mathrm{k}_{\mathrm{B}=-1.11}(7) \mathrm{K}$ for the magnetic layer ' 2 '. We note that the fitting procedure for the bilayer (bpdc-based) material remains discriminant with these five parameters (less parametrized fits result in significantly worse fits). Moreover, the values of the exchange parameter $J_{1}$ for the magnetic layer ' 1 ' are reasonably close in the bdc- and bpdc-based materials. Finally, the constants that are extracted for layer ' 2 ' are reasonable: the exchange through the carboxylate bridges, $J_{2}$, is similar to that in layer ' 1 ', and the interchain coupling $J 2$ ' is significantly weaker, as expected from the larger intermetallic distance.

Whilst the single-layer (bdc-based) material is known to order antiferromagnetically below $T_{\mathrm{N}} \approx 6.5 \mathrm{~K}^{12 \mathrm{~b}}$ our set of macroscopic measurements (see Supporting Information) reveals a more complex behavior in the bilayer material below $T=5 \mathrm{~K}$. Both magnetic susceptibility and heat capacity measurements indicate the existence of multiple magnetic phase transitions, as expected given the intricate crystal chemistry of the material. We observe two transitions upon decreasing temperature, at $T_{\mathrm{N}_{1}} \approx 4.3 \mathrm{~K}$ and $T_{\mathrm{N}_{2}} \approx 4.0 \mathrm{~K}$, which likely correspond to distinct 3-dimensional antiferromagnetic orderings of layers ' 1 ' and layers '2', respectively. Further cooling reveals a third magnetic transition, at $T_{\mathrm{t}} \approx 3.4 \mathrm{~K}$, that features a weak ferromagnetic character. This last transition, absent in the bdc-based material, may be due to a slight canting in layer ' 2 ', where $\mathrm{Mn}^{2+}$ is five-coordinated and therefore more likely subject to a weak single-ion anisotropy.
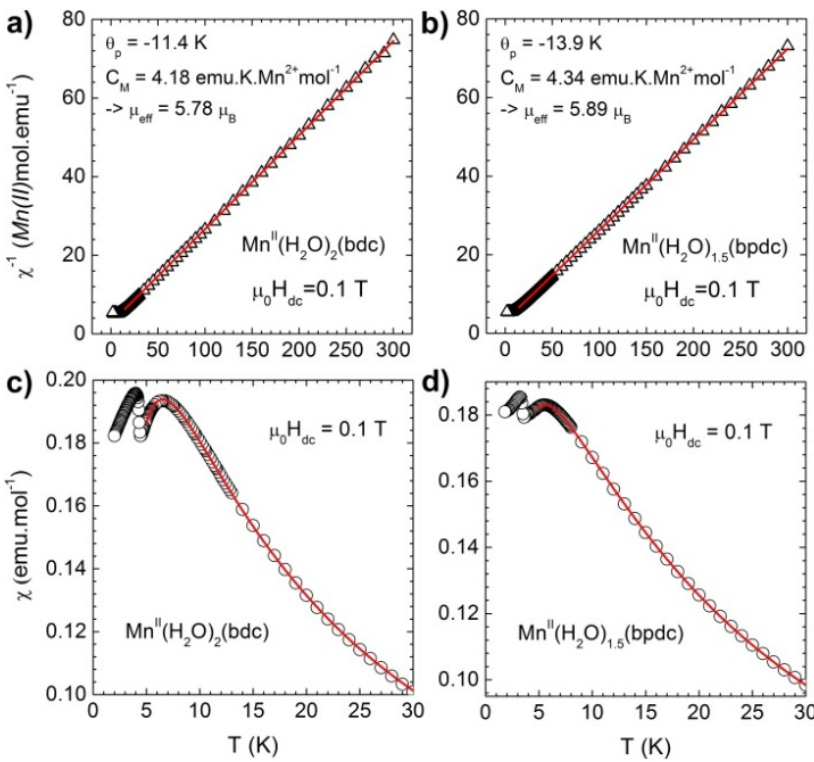

Figure 3. Temperature dependence of the magnetic susceptibility for $\mathrm{Mn}\left(\mathrm{H}_{2} \mathrm{O}\right)_{x}\left(\mathrm{OOC}-\left(\mathrm{C}_{6} \mathrm{H}_{4}\right)_{y}-\mathrm{COO}\right) \mathrm{CNs}$. The magnetic susceptibility $\chi$ (c and $\mathrm{d}$ ) and its inverse value $\chi^{-1}$ (a and $\mathrm{b}$ ) are presented for the bdc- (panels a and c) and bpdc- (panels b and d) based materials. The red lines ( $a$ and b) show the best fits to the Curie-Weiss law in the temperature range between 15 and $300 \mathrm{~K}$. The red curves ( $\mathrm{c}$ and $\mathrm{d}$ ) show the best fits to the exchange interactions' models (see text and Figure 2) in the temperature range from 5 to $30 \mathrm{~K}$.

In conclusion, different network architectures were obtained during the auto-assembly in water of $\mathrm{Mn}^{2+}$ cations and the aromatic dicarboxylate linkers bdc or bpdc. This appears to be distinct from the reticular chemistry approach, ${ }^{1}$ where such organic ligands typically maintain the topology of the resulting MOFs. ${ }^{18}$ Remarkably, the change of the organic ligand from bdc to bpdc appears to trigger the formation of a second type of inorganic subnet in $\mathrm{Mn}\left(\mathrm{H}_{2} \mathrm{O}\right)_{1.5}(\mathrm{bpdc})$. Although it is relatively widespread to find different types of cluster-like Secondary Building Units (SBUs) in MOFs, ${ }^{\text {10a }}$ the coexistence of cluster-like SBUs and extended SBUs (such as rods or layers $)^{10 b, 19}$ - or even more the coexistence of two types of extended SBUs ${ }^{10 b, 11 a, 20}$ - is rarely observed in the same structure. The layered organization found in $\mathrm{Mn}\left(\mathrm{H}_{2} \mathrm{O}\right)_{1.5}(\mathrm{bpdc})$ is most naturally compared with the structures of inorganic materials derived from perovskite blocks, as already pointed out in the study of $\mathrm{Mn}\left(\mathrm{C}_{4} \mathrm{H}_{4} \mathrm{O}_{4}\right){ }^{11 \mathrm{a}}$ The system formed by $\mathrm{Mn}\left(\mathrm{H}_{2} \mathrm{O}\right)_{2}$ (bdc) and $\mathrm{Mn}\left(\mathrm{H}_{2} \mathrm{O}\right)_{1.5}($ bpdc $) \mathrm{CNs}$ appears remarkable in the way the structure reacts to the change of ligand. The substitution of bdc for bpdc in $\mathrm{Mn}^{2+}$ layered hydrates disrupts the sequence of organic and inorganic slabs along the stacking direction, while the crystal chemistry is partly conserved in the plane, where the metrics remains nearly unchanged. Our results further demonstrate the analogy between CNs, such as for instance MOFs, and nanostructures, in which different types of inorganic materials are used to tailor functional magnetic properties.

\section{ASSOCIATED CONTENT}

\section{Supporting Information}

Crystallographic data, infrared spectra and additional physical measurements and fitting details. This material is available free of charge via the Internet at http://pubs.acs.org. 


\section{AUTHOR INFORMATION}

\section{Corresponding Author}

romain.sibille@psi.ch

\section{ACKNOWLEDGMENT}

We thank the SLS (Villigen, Switzerland) and SOLEIL (Saint Aubain, France) synchrotrons for the provision of research facilities. E. Elkaïm, our local contact at the CRISTAL beamline (SOLEIL), is warmly acknowledged. We thank B. Monod and L. Aranda for infrared and TGA measurements, respectively. R. S. thanks M. Kenzelmann, Ch. Rüegg and E. Lhotel for valuable discussions. We acknowledge funding from the Swiss National Science Foundation (R'Equip project 206021_139082 and Fellowship No. P2EZP2_175092). This work used resources of the National Synchrotron Light Source II, a U.S. Department of Energy (DOE) Office of Science User Facility operated for the DOE Office of Science by Brookhaven National Laboratory under Contract No. DESCoo12704.

\section{REFERENCES}

(1) (a) Yaghi, O. M.; Li, G.; Li, H. Selective binding and removal of guests in a microporous metal-organic framework. Nature 1995, 378, 703. (b) Yaghi, O. M.; O'Keeffe, M.; Ockwig, N. W.; Chae, H. K.; Eddaoudi, M.; Kim, J. Reticular synthesis and the design of new materials. Nature 2003, 423, 705. (c) Furukawa, H.; Cordova, K. E.; O'Keeffe, M.; Yaghi, O. M. The Chemistry and Applications of MetalOrganic Frameworks. Science 2013, 341, 974

(2) (a) Kurmoo, M. Magnetic Metal-Organic Frameworks. Chem. Soc. Rev. 2009, 38, 1353. (b) Dechambenoit, P.; Long, J. R. Microporous magnets. Chem. Soc. Rev. 2011, 40, 3249. (c) Maspoch, D.; Ruiz-Molina, D.; Veciana, J. Magnetic nanoporous coordination polymers. J. Mater. Chem. 2004, 14, 2713. (d) Rao, C. N. R.; Cheetham, A. K.; Thirumurugan, A. Hybrid inorganic-organic materials: a new family in condensed matter physics. J. Phys. Condens. Matter 2008, 20, 159801. (e) Minguez Espallargas, G.; Coronado, E. Magnetic functionalities in MOFs: from the framework to the pore. Chem. Soc. Rev. 2018, 47, 533.

(3) (a) Wang, C.; Liu, D.; Lin, W. Metal-Organic Frameworks as A Tunable Platform for Designing Functional Molecular Materials. J. Am. Chem. Soc. 2013, 135, 13222-13234. (b) Clemente-Leon, M.; Coronado, E.; Marti-Gastaldo, C.; Romero, F. M. Multifunctionality in hybrid magnetic materials based on bimetallic oxalate complexes. Chem. Soc. Rev. 2011, 40, 473-497. (c) Delahaye, E.; Eyele-Mezui, S.; Diop, M.; Leuvrey, C.; Foix, D.; Gonbeau, D.; Rabu, P.; Rogez, G. Functional Heterometallic Layered Hybrid Magnets by Double IonExchange. Eur. J. Inorg. Chem. 2012, 16, 2731-2740.

(4) (a) Abellan, G.; Marti-Gastaldo, C.; Ribera, A.; Coronado, E. Hybrid Materials Based on Magnetic Layered Double Hydroxides: A Molecular Perspective. Acc. Chem. Res. 2015, 48, 1601-1611. (b) Rogez, G.; Massobrio, C.; Rabu, P.; Drillon, M. Layered hydroxide hybrid nanostructures: a route to multifunctionality. Chem. Soc. Rev. 2011, 40, 1031-058. (c) Rabu, P. and Drillon, M. (2003) Layered OrganicInorganic Materials: A Way Towards Controllable Magnetism, in Functional Hybrid Materials (eds P. Gómez-Romero and C. Sanchez), Wiley-VCH Verlag GmbH \& Co. KGaA, Weinheim, FRG. (d) Rabu, P., Drillon, M., Awaga, K., Fujita, W. and Sekine, T. (2001) Hybrid Organic-Inorganic Multilayer Compounds: Towards Controllable and/or Switchable Magnets, in Magnetism: Molecules to Materials II: Models and Experiments (eds J. S. Miller and M. Drillon), Wiley-VCH Verlag GmbH \& Co. KGaA, Weinheim, FRG. (e) Pedesseau, L.; Sapori, D.; Traore, B.; Robles, R.; Fang, H-H.; Loi, M. A.; Tsai, H.; Nie, W.; Blancon, J-C.; Neukirch, A.; Tretiak, S.; Mohite, A. D.; Katan, C.; Even, J.; Kepenekian, M. Advances and Promises of Layered Halide Hybrid Perovskite Semiconductors. ACS Nano 2016, 10, 9776-9786. (f) Delahaye, E.; Eyele-Mezui, S.; Bardeau, J-F.;
Leuvrey, C.; Mager, L.; Rabu, P.; Rogez, G. New layered organicinorganic magnets incorporating azo dyes. J. Mater. Chem. 2009, 19, 6106-6115.

(5) Huang, Z-L.; Drillon, M.; Masciocchi, N.; Sironi, A.; Zhao, J-T.; Rabu, P.; Panissod, P. Ab-Initio XRPD Crystal Structure and Giant Hysteretic Effect $\left(H_{\mathrm{c}}=5.9 \mathrm{~T}\right)$ of a New Hybrid Terephthalate-Based Cobalt(II) Magnet Chem. Mater. 20oo, 12, 2805-2812.

(6) Jain, R.; Kabir, K.; Gilroy, J. B.; Mitchell, K. A. R.; Wong, K-c.; Hicks, R. G. High-temperature metal-organic magnets. Nature 2007, 445, 291-294.

(7) Coronado, E.; Galan-Mascaros, J. R.; Gomez-Garcia, C. J.; Laukhin, V. Coexistence of ferromagnetism and metallic conductivity in a molecule-based layered compound. Nature, 200o, 408, 447-449.

(8) Ban, V.; Sibille, R.; Mesbah, A.; Gaudisson, T.; Mazet, T.; François, M. Spin-glass state in $\mathrm{Ni}_{5}(\mathrm{OH})_{6}\left(\mathrm{C}_{n} \mathrm{H}_{2 n-4} \mathrm{O}_{4}\right)_{2}$ metal-organic frameworks $(n=6,8,10,12)$. Solid State Sciences. 2014, 37, 154-163.

(9) (a) Coronado, E.; Minguez Espalargas, G. Dynamic magnetic MOFs. Chem. Soc. Rev. 2013, 42, 1525. (b) Abellan, G.; Jorda, J. L.; Atienzar, P.; Varela, M.; Jaafar, M.; Gomez-Herrero, J.; Zamora, F.; Ribera, A.; Garcia, H.; Coronado, E. Stimuli-responsive hybrid materials: breathing in magnetic layered double hydroxides induced by a thermoresponsive molecule. Chem. Sci. 2015, 6, 1949.

(10) (a) O'Keefe, M.; Yaghi, O. M. Deconstructing the Crystal Structures of Metal-Organic Frameworks and Related Materials into Their Underlying Nets. Chem. Rev. 2012, 112, 675-702. (b) Schoedel, A.; Li, M.; Li. D.; O'Keefe, M.; Yaghi, O. M. Structures of MetalOrganic Frameworks with Rod Secondary Building Units. Chem. Rev. 2016, 116, 12466-12535.

(11) (a) Saines, P. J.; Melot, B. C.; Seshadri, R.; Cheetham, A. K. Synthesis, Structure and Magnetic Phase Transitions of the Manganese Succinate Hybrid Framework, $\mathrm{Mn}\left(\mathrm{C}_{4} \mathrm{H}_{4} \mathrm{O}_{4}\right)$. Chem. Eur. J. 2010 16, 7579-7585. (b) Saines, P. J.; Hester, J. R.; Cheetham, A. K. Neutron diffraction study of the magnetic structures of manganese succinate $\mathrm{Mn}\left(\mathrm{C}_{4} \mathrm{H}_{4} \mathrm{O}_{4}\right)$ : A complex inorganic-organic framework. Phys. Rev. B. 2010, 82, 144435 .

(12) (a) Kaduk, J. A. Terephthalate salts of dipositive cations. Acta Cryst. 2002, B58, 815. (b) Sibille, R.; Mesbah, A.; Mazet, T.; Malaman, B.; Capelli, S.; François, M. Magnetic measurements and neutron diffraction study of layered hybrid compounds $\mathrm{Mn}\left(\mathrm{C}_{8} \mathrm{H}_{4} \mathrm{O}_{4}\right)\left(\mathrm{H}_{2} \mathrm{O}\right)$ and $\mathrm{Mn}_{2}(\mathrm{OH})_{2}\left(\mathrm{C}_{8} \mathrm{H}_{4} \mathrm{O}_{4}\right)$ J. Sol. State Chem. 2012, 186, 134-141.

(13) Neumann, M. A. X-Cell: a novel indexing algorithm for routine tasks and difficult cases. J. Appl. Crystallogr. 2003, 36, 356-365.

(14) Favre-Nicolin, V.; Cerny, R. FOX, 'free objects for crystallography': a modular approach to ab initio structure determination from powder diffraction. J. Appl. Crystallogr. 2002, 35, 734-743.

(15) Rodriguez-Carvajal, J. Recent advances in magnetic structure determination by neutron powder diffraction + FullProf. Physica B. 1993, 55, 192.

(16) (a) Rushbrooke, G. S.; Wood, P. J. On the High-Temperature Susceptibility for the Heisenberg Model of a Ferromagnet. Proc. Phys. Soc. 1995, 68, 1161-1169. (b) Rushbrooke, G. S.; Wood, P. On the Curie points and high temperature susceptibilities of Heisenberg model ferromagnetics. J. Mol. Phys. 1958, 1, 257-283. (c) Stephenson, R. L.; Prinie, K.; Wood, P. J.; Eve, J. On the high temperature susceptibility and specific heat of the Heisenberg ferromagnet for general spin. Phys. Lett. A. 1968, 27, 2-3. (d) Yamaji, K.; Kondo, J. On the High-Temperature Susceptibilities of the Two-Dimensional Ferromagnetic Heisenberg Spin Systems. J. Phys. Soc. Jpn. 1973, 35, 25-32.

(17) Fisher, M. E. Magnetism in One-Dimensional Systems-The Heisenberg Model for Infinite Spin. Am. J. Phys. 1964, 32, 343.

(18) Eddaoudi, M.; Kim, J.; Rosi, N.; Vodak, D.; Wachter, J.; O'Keefe, M.; Yaghi, O. M. Systematic design of pore size and functionality in isoreticular MOFs and their application in methane storage. Science, 2002, 295, 469-472.

(19) (a) Li, Y-W.; Zhao, J-P.; Wang, L-F.; Bu, Y-H. An Fe-based MOF constructed from paddle-wheel and rod-shaped SBUs involved in situ generated acetate. CrystEng Comm, 2011, 13, 6002-60o6. (b) Liu, B.; Liu, B.; Pang, L.; Cui, L.; Wang, Y-Y.; Shi, Q. Unprecedented ${ }_{1} \mathrm{D}$ alternate $\mathrm{Co}_{5}$ chain and discrete $\mathrm{Co}_{3}$ unit embedded in a $3 \mathrm{D}$ framework exhibiting slow magnetic relaxation behavior. CrystEngComm, 2013, 15, 5205-5208. 
(20) Song, Y.; Yin, X.; Tu, B.; Pang, Q.; Li, H.; Ren, X.; Wang, B.; Li, Q. Metal-organic frameworks constructed from mixed infinite inorganic units and adenine. CrystEngComm, 2014, 16, 3082-3085.

SYNOPSIS TOC. $\mathrm{Mn}\left(\mathrm{H}_{2} \mathrm{O}\right)_{15}\left(\mathrm{OOC}-\left(\mathrm{C}_{6} \mathrm{H}_{4}\right)_{2}-\mathrm{COO}\right)$ is a rare example of a layered Coordination Network $(\mathrm{CN})$ containing two types of extended (2D) Secondary Building Units (SBUs). One of the extended SBUs is also found in $\mathrm{Mn}\left(\mathrm{H}_{2} \mathrm{O}\right)_{2}\left(\mathrm{OOC}-\left(\mathrm{C}_{6} \mathrm{H}_{4}\right)_{1}-\mathrm{COO}\right)$, obtained using the same synthesis procedure. Therefore, the change of organic ligand controls the stacking of different types of magnetic layers. This paradigm to prepare materials with tailored magnetic properties further demonstrates the analogy between magnetic CNs and nanostructures made of thin films of different materials.

\section{Table Of Content artwork}

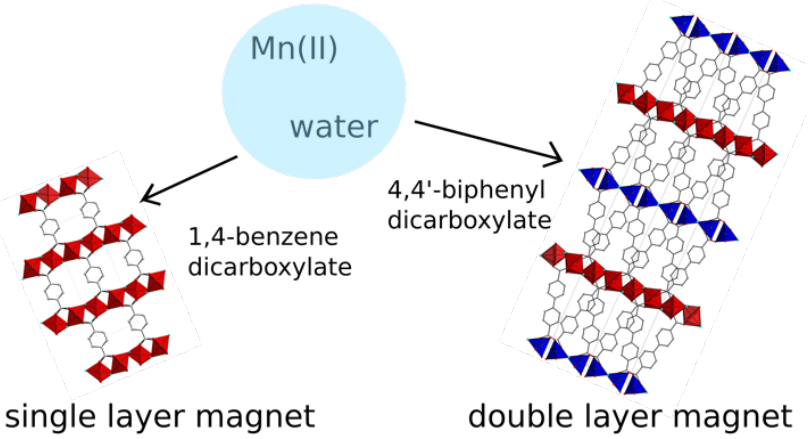

